\title{
Virtualidad en la educación: el desafío de construir calidad, igualdad y equidad en la escuela en tiempos de pandemia
}

\author{
Virtuality in education: the challenge of building quality, equality and \\ equity at schools in times of pandemic
}

\section{${ }^{* 1}$ Liliana Beatriz Barrionuevo, ${ }^{* 2}$ Silvia del Carmen Boccardo \& $* 3$ Silvia Gladys Medina}

Barrionuevo, L., Boccardo, S., \& Medina, S. (2021). Virtualidad en la educación: el desafío de construir calidad, igualdad y equidad en la escuela en tiempos de pandemia. Revista Convergencia Educativa, (10), diciembre, 44-54. http://doi.org/10.29035/rce.10.44

[Recibido: 30 septiembre, 2021 / Aceptado: 28 octubre, 2021]

\section{RESUMEN}

El presente escrito analiza los desafíos que los estados latinoamericanos y en especial Argentina han afrontado y deben afrontar frente reto de construir calidad, igualdad y equidad en la escuela atravesada por la pandemia de Covid-19 en los años 2020 y 2021. La metodología responde al análisis de narraciones de docentes y datos que se obtuvieron sobre capacitaciones a supervisores y profesores de educación secundaria y superior no universitaria y universitaria, frente a la pandemia en instituciones de distintos niveles y de gestión estatal y privada, en el período 2020 - 2021 . El análisis gira desde la reflexividad a partir de la teoría la cual nos permite indagar y establecer criterios de perspectivas que conforman la situación para poder aproximarnos a conclusiones, que no son definitivas, pero que permiten entrever el futuro.

Palabras clave: instituciones escolares, calidad educativa, distanciamiento, políticas educativas, oportunidades de aprendizajes.

\footnotetext{
* Doctoranda del Doctorado en Educación de la Universidad Católica de Córdoba (Universidad Jesuita) - Argentina

1 Profesora de inglés para el nivel primario y secundario. Universidad Nacional de Córdoba, Argentina. https://orcid.org/0000-0002-6070-1525 | misslilibarrionuevo@yahoo.com.ar

2 Especialista en Ciencias Sociales con Mención en Currículum y Prácticas Escolares, FLACSO. Santa Fe, Argentina. https://orcid.org/0000-0002-4655-5699 | silviacboccardo@gmail.com

${ }^{3}$ Licenciada en Ciencias de la Educación. Universidad Católica de Córdoba. Córdoba, Argentina.
} https://orcid.org/0000-0002-9189-7305 | silgmed@gmail.com 
Barrionuevo, L., Boccardo, S., \& Medina, S. (2021). Virtualidad en la educación: el desafío de construir calidad, igualdad y equidad en la escuela en tiempos de pandemia. Revista Convergencia Educativa, (10), diciembre, 44-54. http://doi.org/10.29035/rce.10.44

\section{ABSTRACT}

This article analyzes the challenges that de latinamerican states, and specially Argentina, have faced and must go on doing so, as regards the building of quality, equality and equity in schools affected by Covid-19 in 2020 and 2021 . The methodology responds to the analysis of teachers' narrations and data obtained from training to supervisors and teachers of high-school and higher education (university and non-university), in the context of the pandemic in educational institutions of different levels as well as state and private schools. The analysis is based on the reflexivity theory, which allows us to investigate and establish criteria of perspectives that shape the situation in order reach conclusions that are not definitive, yet allow us to get a glimpse of the future.

Key words: educational institutions, educational quality, lockdown, educational policies, learning opportunities.

\section{INTRODUCCIÓN}

A fines de abril se cumplió un año del mayor cierre de escuelas de la historia moderna. Más del 90\% de las y los estudiantes en 190 países del mundo vieron suspendida la educación presencial. Luego de las respuestas de emergencia, especialistas se preguntan cuánto tiempo tomará la recuperación educativa, cuáles son las medidas prioritarias y qué recursos demandan (Matovich \& Cardini, 2021). La pandemia nos encontró a todos en una situación sin precedentes, y se presenta como "un corte inesperado" que sin dudas modificará las imágenes educativas de los próximos años y décadas (Matovich \& Cardini, 2021). El mundo, nuestra región y nuestro país, se sumieron en un masivo traslado de las actividades a los hogares, especialmente aquellas que podían ser realizadas desde la distancia para evitar la propagación del virus y mitigar su impacto. Esto obligó a contemplar las diferencias que los contextos familiares suponen frente a las posibilidades tecnológicas. En el ámbito educativo las medidas tomadas en políticas por los países de la región, expresa la Comisión Económica para América Latina y el Caribe [CEPAL] (2020) que giraron en torno a la suspensión de clases de la modalidad presencial, en todos los niveles (primario, secundario, superior no universitario, universitario), lo que originó diferentes acciones en lo referente al ingreso del aprendizaje a distancia, a través de internet, activando a las diversas comunidades educativas y procurando afianzar el vínculo afectivo entre los diferentes agentes educativos.

Asimismo, en nuestro país, desde los diferentes estamentos gubernamentales, se adecuaron plataformas virtuales de contenidos que ya existían, como por ejemplo, "Educ.ar", se lanzó una línea educativa en WhatsApp, se entregaron licencias a docentes y estudiantes de escuelas técnicas para realizar prácticas virtuales en laboratorios de Física, Química, Matemática, etc.; propuestas de formación y capacitación docente; se puso a disposición material impreso para la población con impedimentos al acceso a medios digitales; se abordaron estrategias enfocadas a transferir recursos de aprendizajes hacia docentes $\mathrm{y}$ familias; se re-organizaron los contenidos y metas de aprendizaje, se implementó un reordenamiento temporal del año escolar, teniendo en cuenta una progresión de aprendizaje que abarca el 2020 y 2021 y se puso en marcha una evaluación expresada en informes descriptivos y conceptuales. 
Barrionuevo, L., Boccardo, S., \& Medina, S. (2021). Virtualidad en la educación: el desafío de construir calidad, igualdad y equidad en la escuela en tiempos de pandemia. Revista Convergencia Educativa, (10), diciembre, 44-54. http://doi.org/10.29035/rce.10.44

Esta situación de emergencia sanitaria tiene y tendrá importantes efectos negativos y positivos en los distintos sectores sociales, y el campo de la educación no es la excepción. Lewin (2020) sostiene que una cosa quedó clara y ha sido que el sistema educativo argentino no estaba preparado para garantizar una educación virtual igualitaria y a distancia.

Ante este panorama nos preguntamos: ¿cuáles han sido los mecanismos que los distintos gobiernos de la región pusieron en marcha para enfrentar esta situación problemática del Covid-19, promoviendo oportunidades de aprendizajes para todos/as y garantizar el derecho a una educación de calidad, igualitaria y equitativa?

El presente trabajo tiene por objetivo "abordar los nuevos modos que la intervención docente en la clase demanda, a partir de la irrupción de la virtualidad en el aula, y en los distintos niveles del sistema; los diversos mecanismos que se pusieron en marcha para afrontar el desafío de garantizar el derecho a una educación de calidad, igualitaria y equitativa, promoviendo oportunidades de aprendizaje para todos". Se considera necesario profundizar desde diferentes perspectivas, el abordaje de las complejas realidades de los/as estudiantes, en un contexto donde la virtualidad se instaló, en entornos donde la conectividad no era posible, teniendo que acudir a la implementación de cuadernillos, guías de aprendizajes y de actividades, como modalidad para sostener el vínculo pedagógico en el proceso de enseñanza y de aprendizaje.

La determinación de los gobiernos estatales de suspender las actividades presenciales y el cierre de los establecimientos escolares, marcó una brecha significativa en lo educativo. Dada la primera ola global del coronavirus (COVID-19), y a fin de mitigar el impacto del cierre de las escuelas, se desarrollaron iniciativas para asegurar la continuidad del proceso de aprendizaje, y para ello se enfocó en la introducción o ampliación de la modalidad de aprendizaje a distancia o remoto, a través de entornos digitales y plataformas web. Esto significó que los distintos países, tanto a nivel regional como internacional, recurrieran al uso masivo de internet. Esta propuesta "de profundas transformaciones e innovaciones" intentó convertirse en una garantía para el acceso a la educación, sin embargo, diferentes factores profundizaron la inequidad, ya existente, al acceso a una educación de calidad (Bonilla, 2020, p. 90). Una de las dificultades fue la falta de recursos tecnológicos, tanto de estudiantes como de docentes, que este nuevo mecanismo requiere para la escolarización. Asimismo, se percibió una carencia de habilidades específicas por parte de los estudiantes para hacer frente a los requerimientos que un entorno virtual exige, como también la falta de preparación y experiencia por parte de los docentes, para desarrollar estrategias de enseñanza y de aprendizaje remotos. Así se instaló un escenario de inseguridades e incertidumbres que afectó directamente el proceso, y que vulneró, en cierto modo, la calidad educativa y que se apreció a lo largo de este período de confinamiento y bimodalidad educativa.

De esta manera, la modalidad virtual hizo más evidente la exclusión y desigualdad que las instituciones educativas han cobijado en todas las épocas. Un mundo en donde algunos alumnos podían continuar sus estudios en entornos virtuales, respondiendo a las demandas que esta modalidad implica; y otros, que 
Barrionuevo, L., Boccardo, S., \& Medina, S. (2021). Virtualidad en la educación: el desafío de construir calidad, igualdad y equidad en la escuela en tiempos de pandemia. Revista Convergencia Educativa, (10), diciembre, 44-54. http://doi.org/10.29035/rce.10.44

acorde a sus posibilidades, hicieron frente a esta situación con notables desventajas. La reapertura de escuelas en 2021 estuvo, también, acompañada de políticas de organización en tres maneras de transitar la educación obligatoria: presencial, no presencial y combinada. Esta organización demanda nuevos saberes, un nuevo currículum y nuevas formas de gestionar las clases.

La educación a distancia y/o por medios digitales, nos hizo repensar qué contenidos son prioritarios, cómo se evalúa, cómo sostener los vínculos pedagógicos, entre otras cuestiones. Las autoridades gubernamentales, los equipos directivos y los profesores de diferentes niveles se encontraron ante un cambio que el ASPO y DISPO ${ }^{1}$ nos había presentado.

Ante esta situación surgen una serie de interrogantes ¿Los actores institucionales estuvimos y estamos formados para afrontar dichos desafíos? ¿Pudimos distanciarnos de las pedagogías estructuradas o volvimos a propuestas poco constructivistas? ¿Cómo afrontamos este nuevo camino de la educación y cómo lo vamos a continuar?

\section{DESARROLLO}

Estos momentos históricos, donde se nos invita a ser protagonistas, líderes y transformadores, constituyen un enorme desafío, pero con certeza de que serán también los logros de todos los actores involucrados. La pandemia provocó cambios sin precedentes en todos los ámbitos. Rupturas que tienen y tendrán importantes efectos en los distintos sectores sociales, pero que, en el campo de las políticas educativas, significó y significa múltiples desafíos para garantizar el derecho a la educación en condiciones de amplia desigualdad e incertidumbre.

El "distanciamiento social, preventivo y obligatorio" que se estableció para evitar el riesgo de propagación del virus SARS-CoV-2 para proteger la salud pública de la población

Este momento significó, por un lado, que las instituciones educativas dejaron de ser el lugar donde se aprende y se trasladaron al hogar. Las condiciones habitacionales, el acceso a dispositivos tecnológicos y el capital educativo de los hogares son factores que se manifiestan de forma diversa en nuestro territorio nacional y esto marcó "el volver a pensar" que el mundo educativo ya estaba inmersos en dos tipos de distanciamiento por la fragmentación que existe en la sociedad e impacta en las instituciones educativas: un distanciamiento físico que era evidente pero no asumido en las escuelas por los profesores y un distanciamiento social que impacta en la exclusión de nuestros/as estudiantes.

Pensamos la fragmentación y la falta de equidad como una "frontera de referencia", sin constituir un todo integrado y coordinado y sí como una "ruptura"; situaciones que permiten visualizar en el proceso de

1 ASPO implica el Aislamiento Social, Preventivo y Obligatorio para la primera etapa de la pandemia, mientras que el DISPO refiere al Distanciamiento Social, Preventivo y Obligatorio en la segunda etapa pandémica. 
Barrionuevo, L., Boccardo, S., \& Medina, S. (2021). Virtualidad en la educación: el desafío de construir calidad, igualdad y equidad en la escuela en tiempos de pandemia. Revista Convergencia Educativa, (10), diciembre, 44-54. http://doi.org/10.29035/rce.10.44

enseñar y de aprender nuevas dificultades para supervisores (inspectores), profesores, estudiantes y familias que, en el día a día de la educación, se fueron y se siguen naturalizando, las prácticas educativas se vieron y se ven afectadas por este distanciamiento físico y social que se hizo aún más presente en la virtualidad, porque las prácticas institucionales y áulicas ya pertenecían a un sistema en conflicto, donde no se encontraban las formas o las estrategias para seguir hacia un futuro esperado, ocultando la necesidad de una transformación. Esta situación hace que en el espacio interior se puedan distinguir continuidades y diferencias, o hasta una suma de fragmentos cerrados o con escasa articulación. Además, esta idea es una de las razones que nos permite volver a mirar la configuración actual del sistema educativo y revisar la escuela y las universidades como instituciones donde se forjan la formación de subjetividades en un sentido cultural, normativo y social que sistematizan las relaciones e interrelaciones entre los sujetos.

La educación (en todos sus niveles) no ha quedado al margen del impacto del distanciamiento sanitario y social, de la "fragmentación educativa" en la que veníamos inmersos, profundizándose durante los años 2020 y 2021 ¿Qué situaciones o acciones nos permiten volver a pensar la educación de los ciudadanos del futuro? Un camino para la formación general de la ciudadanía sería si las políticas educativas nacionales y provinciales contemplan variedad de aspectos a través de programas de inclusión, calidad y equidad de currículos adaptados a los diferentes niveles con variedad de formatos y tiempos. De esta manera, la modalidad virtual hizo más evidente la exclusión y desigualdad que las instituciones educativas han cobijado en todas las épocas. Sin embargo, cabe interrogar ¿por qué las escuelas y formación superior universitaria y no universitaria siguen expulsando a tantos niños/as, adolescentes, y jóvenes o permiten que ellos solos se excluyan de este espacio de desarrollo personal y grupal? ¿Cuáles son las dificultades que la educación conserva para no poder acompañar e incluir a "todos/as" dentro de las instituciones brindando una educación de calidad? ¿El distanciamiento que se fue profundizando ha sido físico, social, y/o cultural? Estas y otras preguntas ya estaban instaladas previas a la pandemia y a las decisiones de la política educativa y hoy siguen preocupando a la sociedad actual.

Este momento donde fue y es necesario (casi una exigencia) implementar nuevos formatos de gestionar la enseñanza y el aprendizaje, nos invita a revisar, reflexionar y repensar el currículo, junto a una curación de contenidos sobre los diseños de cada nivel educativo, que permite una adaptación a las diferentes necesidades pedagógicas que nos atraviesan como una comunidad educativa en un mundo global. Repensar los contenidos, implica revisar las metodologías de enseñanza y de aprendizaje, y poner en práctica diferentes formas de evaluar y valorar lo enseñado y lo aprendido.

La situación social e histórica actual nos interpela para volver a pensar y replantear los contenidos a desarrollar desde el aislamiento y en los formatos presenciales, semipresenciales y a distancia que aún conviven en las instituciones escolares y de nivel superior. Estudiantes con escasos aprendizajes, con dificultades para interpretar textos $\mathrm{u}$ otros lenguajes nos exigen nuevos y diversos modos y formatos innovadores a partir de contenidos significativos, relevantes y abordados con calidad, que están 
Barrionuevo, L., Boccardo, S., \& Medina, S. (2021). Virtualidad en la educación: el desafío de construir calidad, igualdad y equidad en la escuela en tiempos de pandemia. Revista Convergencia Educativa, (10), diciembre, 44-54. http://doi.org/10.29035/rce.10.44

atravesados por la interdisciplinariedad, y que deben incluir a los/as estudiantes y sus culturas a través de diferentes propuestas de aprender dentro y fuera del aula. Estos contenidos no solo abordan conocimientos disciplinares, hoy nos enfrentamos a la "importancia" que tiene el desarrollo de competencias y macro habilidades que sean transversales a todas las disciplinas, que permitan pensar de manera flexible, divergente y creativa al "otro", que abran puertas para crear y comunicar de manera responsable la construcción y reconstrucción de conocimientos y saberes propios, y de manera prioritaria, que atiendan a la toma de decisiones, al diálogo y a la comunicación permanente a través de buenas preguntas donde juega la creatividad y la reflexión del/la profesor/a y los/as estudiantes.

Otro de los desafíos o quiebres frente a las clases a distancia y virtuales, la selección de contenidos prioritarios y significativos para estos nuevos modos y formatos del proceso de enseñar y aprender fue y es cómo se evalúa o valora el proceso de aprendizaje. La evaluación y acreditación entró en el cuestionamiento de ¿continuidad o ruptura? Lo vivenciado durante los años 2020 y lo transcurrido durante 2021 con el Covid-19 "acentuó muchos temores más o menos infundados" (Chartier, 2021, p.62) acerca del proceso de enseñanza y aprendizaje. Las dudas y la incertidumbre de cómo evaluar y acreditar en los diferentes niveles de educación, las formas de valorar los procesos, la falta de la evaluación cuantitativa se convirtió en un momento de formatos diferentes los cuales implicaron repensar y revisar desde un periodo inédito a través "de un todo digital (o casi) para las comunidades formales e informales" (Chartier, 2021, p. 62) de educación. Esta revisión y reflexión frente a la distancia social y la necesidad de mantener un vínculo afectivo y pedagógico nos exigió ver la evaluación y acreditación de los estudiantes como un proceso de valoración y de continuidad, tarea que nos condujo a la lectura y análisis que nos permitan evolucionar de manera conjunta y colaborativa con el otro al momento de valorar el proceso de construcción de conocimiento y saberes que no sólo pertenecen a los/as estudiantes, sino que el/la profesor/a es, asimismo, parte de dicho acontecimiento educativo, social y cultural.

Hoy, en el año 2021, en el territorio argentino se ha vuelto a la presencialidad y con esta presencia física, donde tal vez perdura la ausencia social, la evaluación pasó a ser un trámite más, un momento de pruebas tradicionales, ¿es este un momento de volver hacia atrás o dar pasos gigantes de transformaciones? Quizás se necesiten de innovaciones que generen y adelanten un futuro educativo que nos permita pensar y reflexionar de manera creativa y autónoma con proyectos de enseñanza y aprendizaje colaborativos a través de medios digitales y tecnológicos que fortalezcan la presencialidad.

Hemos referido que una de las medidas llevadas a cabo por los gobiernos de la región, ante el avance de la pandemia, fue la suspensión de la presencialidad en los establecimientos educativos de todos los niveles, lo que llevó a una alternativa de solución que fue la implementación de la modalidad a distancia, involucrando la adaptación de enfoques orientados al uso de las TIC, lo que planteó interrogantes desde diferentes perspectivas: la del/la docente sobre la preparación y el conocimiento en el uso de estas 
Barrionuevo, L., Boccardo, S., \& Medina, S. (2021). Virtualidad en la educación: el desafío de construir calidad, igualdad y equidad en la escuela en tiempos de pandemia. Revista Convergencia Educativa, (10), diciembre, 44-54. http://doi.org/10.29035/rce.10.44

herramientas digitales, y los modos en que se desarrollaron las actividades de enseñanza y de aprendizaje bajo la modalidad a distancia.

En el aula, los/as docentes a través de sus competencias pedagógicas suelen tener dificultades para atender a la diversidad, en medio de la crisis sanitaria el/la docente se ha visto en la necesidad de aprender a involucrarse en la sociedad del conocimiento de las TIC para no verse ajeno a la realidad digital, con la finalidad de superar las barreras tecnológicas y de conectividad, afirman Macías et al. (2021). Esto nos remite a la competencia digital que involucra el uso creativo, crítico y seguro de las TIC, para alcanzar los objetivos relacionados con el aprendizaje (en nuestro caso), el uso del tiempo, la inclusión y participación en lo educativo. Ahora bien, desde la perspectiva docente la preparación y el conocimiento previo en el uso de recursos y herramientas digitales para desarrollar una clase no presencial nos hace preguntarnos ¿cómo ha llevado la actividad de enseñar bajo la modalidad virtual? Investigadores como Martínez \& Garcés (2020) consideran que la educación en este tiempo de pandemia ha pasado por tres desafíos: la accesibilidad a internet, el uso de herramientas tecnológicas y el acompañamiento de las familias para lograr la continuidad en la formación de los/as estudiantes.

Los principales indicadores, acorde a la Encuesta permanente de hogares (2020), en Argentina registraron en el cuarto trimestre del 2020, que el 63,8\% de los hogares urbanos tienen acceso a computadoras, y el 90\% a internet, mientras que 88 de cada 100 personas emplean teléfono celular y 85 de cada 100 utilizan internet. Estos datos nos permiten tener una idea aproximada sobre cómo los/as estudiantes han resuelto las clases a distancia que tuvieron durante el 2020 y qué acciones llevaron a cabo las instituciones cuando los/as educandos/as informaban a las instituciones escolares el carecer de conectividad. Esto significó que el Ministerio de Educación Nacional, como de las distintas jurisdicciones, tuvieran que elaborar cuadernillos (en el nivel primario y secundario), sobre los ejes prioritarios de enseñanza y de actividades a las que los/as estudiantes resolvían para dar cuenta de sus aprendizajes. Aproximadamente el $78.8 \%$ de los/as escolares pudieron conectarse para seguir las clases virtuales sincrónicas y de modo asincrónicas en estos dos niveles del sistema educativo. Estos datos que reflejan una realidad de inequidad de acceso a posibles modalidades no presenciales nos conducen a considerar que la conectividad y el uso de herramientas digitales han sido y son cruciales en la educación a distancia o remota, y que la democratización del acceso debe estar garantizada por el Estado sin distinción alguna.

En el nivel superior no universitario y universitario, las actividades de enseñanza se resolvían a través de las plataformas con las que contaban, las cuales, en muchos casos, habían sido creadas como apoyo en el dictado de clases, para material complementario y el dictado de cursos online. De esta manera, en este nivel la situación fue menos angustiante, ya que se facilitaron, a modo de comodato elementos tecnológicos para aquellos/as estudiantes que no contaban con los mismos, y de este modo pudieron regularizar el cursado y ser evaluados bajo la modalidad virtual. 
Barrionuevo, L., Boccardo, S., \& Medina, S. (2021). Virtualidad en la educación: el desafío de construir calidad, igualdad y equidad en la escuela en tiempos de pandemia. Revista Convergencia Educativa, (10), diciembre, 44-54. http://doi.org/10.29035/rce.10.44

Finalmente cabe referir al principal actor sin el cual el hecho educativo no tiene sentido y es el/la 'educando' cuyo rol en la virtualidad supone una implicación personal mayor que la requerida en el entorno presencial, que se refleja en una actitud reflexiva hacia su propio proceso de aprendizaje seguido de interrogantes como: “¿qué quiero aprender?, ¿por qué? y ¿para qué?, de acuerdo al nivel educativo en el que se halle, y que deben ser impulsados por cada docente, a partir de estrategias metodológicas que apunten al desarrollo de capacidades de metacognición, de autorregulación, de interacción con el grupo en actividades colectivas. Se debe reforzar en el/la estudiante una disposición particular para aprender en un entorno virtual, apuntando a la autonomía y al aprendizaje cooperativo, siempre vinculando, especialmente en la bimodalidad, los saberes propios del entorno virtual con aquellos del entorno real (Sánchez \& Alvarenga, 2014). De esta manera, los tiempos de la pandemia reconfiguró elementos que están presentes al momento de educar en las sociedades contemporáneas: tiempo, espacio, saberes, valores, transmisión (Dussel et al., 2020).

\section{CONCLUSIÓN}

En el contexto de la emergencia por la pandemia por Covid-19, hemos asistido a un hecho educativo que tuvo que reconfigurarse, en primer lugar el sitio de mayor transformación fueron los hogares de los/as estudiantes y de los/as docentes, escenarios que se metamorfosearon en aulas, oficinas, laboratorios, despachos, etc., donde los padres, madres y a veces hermanos mayores u otros familiares convivientes, desde su experiencia, acompañaron a los/as estudiantes, lo que significó que se revalorizará el rol docente en sus prácticas áulicas, en actividades vinculadas a explicar, organizar y evaluar (Dussel et al. 2020).

La socialización que brinda la presencialidad en las aulas se vio trastocada, mutó a la virtualidad, donde las diferentes ventanas o el solo poder ver al docente impartiendo una clase con diapositivas, con imágenes, con documentales o películas llevó a los/as estudiantes a una construcción deficitaria de la otredad; si bien estaba presente el cara a cara, ¿qué sucedió con aquellos/as que no tuvieron la conectividad óptima o carecían de ella, y lo hicieron a través de cuadernillos? ¿Cómo fue el vínculo pedagógico? Esta situación planteada que pretendía garantizar el proceso educativo e intentaba mantener el vínculo pedagógico a través de la distancia, no logró este objetivo.

Sin embargo, esta virtualidad que irrumpió sorpresivamente tiene aspectos positivos que, al decir de Moreira \& Delgadillo (2015), favorecen aspectos que la presencialidad limita o no contempla: rompe la barrera de la distancia, la rigidez de los horarios y facilita la distribución del tiempo. No obstante, requiere la prevalencia de ciertas estrategias como son la comunicación fluida y efectiva, en una atmósfera cálida y de acompañamiento constante. Ante esta situación, continúan estos autores, en la que se ven inmersos los modelos pedagógicos "lineales, autoritarios y analógicos" se deben favorecer modelos "en red, participativos y digitales en el contexto de procesos educativos dinámicos" (p. 123). Por lo tanto, estos 
Barrionuevo, L., Boccardo, S., \& Medina, S. (2021). Virtualidad en la educación: el desafío de construir calidad, igualdad y equidad en la escuela en tiempos de pandemia. Revista Convergencia Educativa, (10), diciembre, 44-54. http://doi.org/10.29035/rce.10.44

nuevos modelos digitales requieren, por parte del tutor virtual, ciertas características con el objeto de poder guiar y promover un proceso educativo dinámico:

- Ser capaz de crear, adaptar y valorar nuevas actividades que inviten a la reflexión, la crítica y discusión.

- Ser un constante motivador para promover lazos afectivos y reforzar los logros que los estudiantes vayan alcanzando, promoviendo, en todo momento, la participación y la integración y el trabajo autónomo.

- Ser observador del proceso de aprendizaje a través de un constante seguimiento, realimentación y acompañamiento.

- Ser un impulsor de ideas que trasciendan y conecten con la realidad de los participantes logrando así, un aprendizaje significativo: de "aprender a aprender".

- Ser organizado porque el trabajo virtual requiere una dosis extra de planeamiento de recursos y tiempo, como así también una definición de contenidos a trabajar (p. 124).

No obstante, y siguiendo a aquellos autores, la modalidad virtual no solo requiere un ajuste en contenidos, evaluación, roles de los participantes y del tutor, requiere, además, "un pensamiento flexible y holístico" por parte de todos los involucrados que comparten esta modalidad de experiencia educativa. Ciertamente, la experiencia en la educación presencial es importante, pero, no es suficiente para abordar una experiencia en la virtualidad; requiere, esta última, "un cambio de paradigmas y de concepciones con respecto al proceso educativo, acompañado del conocimiento técnico de cómo conducir la virtualidad" (p. 128). Se hace necesario conocer no sólo el ámbito particular del propio espacio curricular, sino tener cierta formación en técnicas didácticas virtuales, junto al uso adecuado de la plataforma en la que su aula virtual, se encuentre. Esta nueva actitud, que trasciende lo meramente educativo, no puede reducirse, solamente, a la capacitación docente, se requiere pensar en recursos que apuesten a la rápida adaptación de los miembros de la comunidad educativa (docentes, estudiantes y familias), a las exigencias de una situación que no brinda el tiempo suficiente para adquirir las habilidades necesarias para enfrentarla. Juega aquí un papel preponderante el Estado, a través de su ministerio de educación, los supervisores de cada institución y su correspondiente equipo directivo.

Desde otra perspectiva, se hace necesario que los/as profesores/as dejen de ser aplicadores de contenidos, y comiencen a ser creadores activos y colaborativos de saberes y habilidades. Ello permitirá poder diseñar procesos de enseñanza-aprendizajes en diferentes entornos y en consecuencia que los/as estudiantes desarrollen las competencias necesarias para asumir desafíos futuros. 
Barrionuevo, L., Boccardo, S., \& Medina, S. (2021). Virtualidad en la educación: el desafío de construir calidad, igualdad y equidad en la escuela en tiempos de pandemia. Revista Convergencia Educativa, (10), diciembre, 44-54. http://doi.org/10.29035/rce.10.44

\section{REFERENCIAS BIBLIOGRÁFICAS}

Comisión Económica para América Latina y el Caribe [CEPAL]. (2020), "América Latina y el Caribe ante la pandemia del COVID-19. Efectos económicos y sociales". Informe Especial COVID-19, (1), 1-15. https://repositorio.cepal.org/handle/11362/45337

Chartier, R. (2021). Lectura y pandemia. Conversaciones. Katz.

Bonilla-Guachamín, J.A. (2020). Las dos caras de la educación en el COVID-19. CienciAmérica, 9(2), 89-98. http://dx.doi.org/10.33210/ca.v9i2.294

Dussel, I., Ferrante, P., \& Pulfer, D. (Comp.). (2020). Nuevas ecuaciones entre educación, sociedad, tecnología y Estado. En Pensar la educación en tiempos de pandemia. Entre la emergencia, el compromiso y la espera. Unipe Uditorial Universitaria. https://articulateusercontent.com/rise/courses/BoJrwDi3AydIzpUmp6oIYmd2CPFA0J4A/xBVQ cdl3PHXJ940b-Dussel-Ferrante-Pulfer-\%2520ecuaciones\%2520-\%2520vf.pdf

Encuesta permanente de hogares. (2020). Microdatos y documentos 2016-2021. https://www.indec.gob.ar/indec/web/Institucional-Indec-BasesDeDatos

Lewin, L. (15 de abril del 2020). La nueva grieta, ahora de la mano de la educación: ¿Escuelas para unos y escuelas para otros? Millones de alumnos sin conectividad o sin dispositivos móviles, se quedaron afuera del sistema. Perfil. https://www.perfil.com/noticias/opinion/opinion-laura-lewincoronavirus-escuela-nueva-grieta-educacion.phtml

Macías-Figueroa, F. M., Mendoza-Vergara, G. M., Mieles-Pico, G. L., \& San Andrés-Soledispa, E. J. (2021). Competencias digitales del docente para atender a la diversidad de aprendizajes en la pandemia COVID-19. Polo del Conocimiento, 6(1), 288-306. https://polodelconocimiento.com/ojs/index.php/es/article/view/2142

Matovich, I., \& Cardini, A. (2021). Educación en América Latina: un futuro que se escribe desde hoy. Redacción Periodismo Humano. https://www.redaccion.com.ar/educacion-en-america-latina-unfuturo-que-se-escribe-desde-hoy/

Martínez-Garcés, J., \& Garcés-Fuenmayor, J. (2020). Competencias digitales docentes y el reto de la educación virtual derivado de la covid-19. Educación y Humanismo, 22(39), 1-16. https://doi.org/10.17081/eduhum.22.39.4114

Moreira, C., \& Delgadillo, B. (2015). La virtualidad en los procesos educativos: reflexiones teóricas sobre su implementación. Tecnología en Marcha, 28(1), 121-129. http://www.scielo.sa.cr/scielo.php?script=sci arttext\&pid=S0379$\underline{39822015000100121 \& \operatorname{lng}=\mathrm{en} \& \ln g=\mathrm{es}}$ 
Barrionuevo, L., Boccardo, S., \& Medina, S. (2021). Virtualidad en la educación: el desafío de construir calidad, igualdad y equidad en la escuela en tiempos de pandemia. Revista Convergencia Educativa, (10), diciembre, 44-54. http://doi.org/10.29035/rce.10.44

Datos de correspondencia

Liliana Beatriz Barrionuevo

Doctoranda del Doctorado en Educación de la

Universidad Católica de Córdoba (Universidad Jesuita) - Argentina

Profesora de inglés para el nivel primario y secundario.

Universidad Nacional de Córdoba

\section{Córdoba, Argentina}

Dirección postal: Dublín 191- B Playas de Oro - CP:5152

Carlos Paz, provincia de Córdoba, Argentina

Research ID: https://orcid.org/0000-0002-6070-1525

Email: misslilibarrionuevo@yahoo.com.ar 